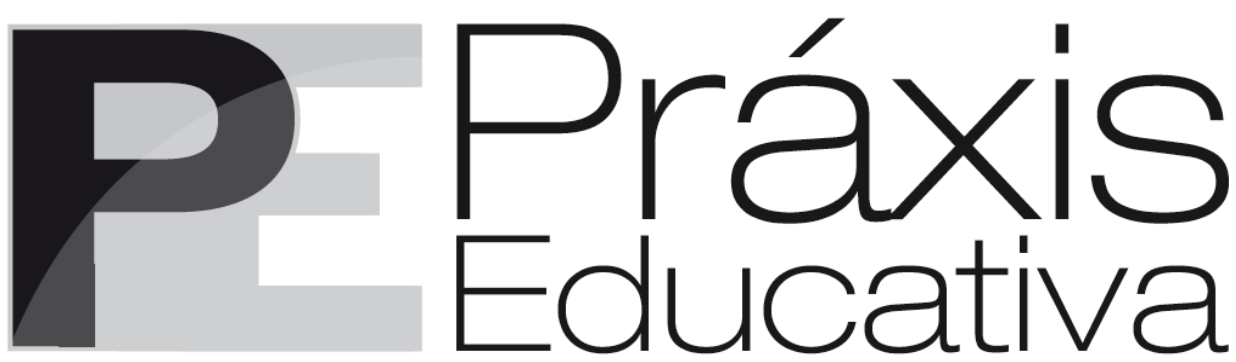

ISSN 1809-4309 (Versão online) DOI: 10.5212/PraxEduc.v.11i2.0015

\title{
PRATS, Enric. La educación, una cuestión de estado. Una mirada a Europa. Barcelona: Edición Universidad de Barcelona, 2013. 227 p.
}

\author{
Rita de Cássia Oliveira Cruz ${ }^{*}$ \\ Remi Castioni* $^{* *}$
}

El panorama actual de cambios en las sociedades de todo el mundo, la globalización y su impacto en las reformas educativas, así como los datos de los informes internacionales respecto al éxito escolar, evidencian la necesidad de ampliar la comprensión sobre estas reformas. En este sentido, el libro de Enric Prats ${ }^{1}$ es una obra relevante para esa cuestión puesto que presenta, entre otros aspectos, un análisis interpretativo de datos del Informe PISA ${ }^{2}$ - Programme for International Students Assessment. El autor centra la atención en un elemento del currículo de la educación básica de los países europeos: la comprensión lectora. Dicha obra constituye un referencial en el ámbito de las políticas educativas, la evaluación y el cambio del currículo para diferentes contextos y países.

La obra de Prats está escrita en catalán y se desdobla en un prólogo y cuatro capítulos. El prólogo evidencia la proposición de una mirada comparativa sobre la educación de países europeos con la intención de analizar precisamente el grado de ajuste del sistema educativo español, y en particular de Cataluña, al "ansia de acercamiento más estrecho con los vecinos europeos” (PRATS, 2013, p. 10). Pues, según el autor, la educación refleja un cruce de intereses, tradiciones y perspectivas muy dispares en el interior de la Unión Europea. Como ejemplo menciona que el gobierno español presentó, en diciembre de 2012, una nueva ley (la décima ley educativa en tres décadas) con fuerte carga ideológica. En este sentido, es necesario reflexionar en torno a los rankings internacionales, puesto que las reformas educativas pasan por alto el debate social y las decisiones políticas no tienen una base empírica sólida y suficientemente contrastada con estudios rigurosos.

En la introducción, se recoge que la filosofía que impregna la obra tiene como base dos puntos de vista: el concepto de educación y la pedagogía como asignatura. Por tanto, el autor desarrolla un comparativo, en base a un contexto permeado por una tensión (entre las tendencias dirigidas a la globalización y las que emanan de las realidades nacionales) y en base a informes de organismos internacionales.

\footnotetext{
*Profesora del Instituto Federal de Goiás/Brasil. E-mail: <rita.cruz@terra.com>.

** Profesor de la Facultad de Educación de la Universidade de Brasília. E-mail: <rcastioni@globo.com>.

${ }^{1}$ Es doctor en Pedagogía por la Universidad de Barcelona y profesor de Pedagogía del Departamento de Teoría e Historia de la Educación de la misma universidad.

2 Acrónimo en inglés del Programa de Evaluación Internacional de los Estudiantes.
} 
PRATS, Enric. La educación, una cuestión de estado. Una mirada a Europa...

Según Prats (2013), la pedagogía comparada, asignatura que anteriormente estaba reservada a un grupo de universidades, pasa a ocupar una posición mediática en la sociedad. Hecho que resultó en investigaciones internacionales sobre el estado de la educación en el mundo, provocando una determinada "opinión pública" o publicada que empieza a demandar explicaciones a las autoridades acerca del desempeño de los estudiantes y de los resultados mediocres en la escuela. También afirma que desde la pedagogía continúa reclamándose que se aborde el tema, considerando una metodología rigorosa y adaptada al tiempo corriente, que lleva en consideración el análisis comparado y el estudio histórico. De hecho, no se puede negar el efecto negativo de la comparación, puesto su uso perverso como una fotocopia en blanco y negro, es decir, se niega la posibilidad de reconocer lo que se está haciendo bien en cada país. Además, menciona la necesidad de buscar los antecedentes catalanes respecto a esta asignatura, puesto que Cataluña posee una importante tradición comparativa (PRATS, 2013, p. 18). Sin embargo, según él, esta región sufre una patología doble: bulimia de datos y anorexia interpretativa.

El primer capítulo del libro, "El estado de la cuestión: resultados y efectos", que se desdobla en tres partes, evidencia cierta preeminencia puesto que presenta relatos comparativos de la educación en Europa. La primera parte ("Niveles de formación") registra que el acceso a la educación básica es prácticamente universal en todos los países desarrollados y que en el sistema educativo europeo hay una diversidad de nomenclaturas, estructuras y límites en las etapas formales de este sistema. Pero, en líneas generales, presenta la siguiente organización: educación preprimaria, es la puerta de entrada al sistema educativo formal y no posee carácter obligatorio en ningún país europeo; educación primaria, en la mayoría de los países es la primera parte de la educación básica y favorece los rudimentos esenciales en lectura, escritura y matemáticas, además de competencias nucleares en otras materias: conocimiento del medio, conocimientos científicos, artísticos, etc. (se sitúa entre los 5 y los 7 años de edad, con una duración aproximada de cinco o seis años); educación secundaria, es la segunda parte de la educación básica y completa la educación obligatoria, su duración se sitúa entre tres y cuatro años al final de la etapa (suele coincidir con la edad legal para la inserción laboral); educación secundaria superior que tiene inicio después de haber superado la educación obligatoria y, en el caso español, las dos opciones reciben, respectivamente, los nombres de bachillerato y formación profesional de grado medio; educación postsecundaria no terciaria (supone un cierto avance respecto a los estudios secundarios pero no tiene el nivel de los estudios superiores o terciarios - suelen ser estudios de especialización en determinadas ramas profesionales $-\mathrm{y}$ no hay equivalencia en el sistema educativo español).

Cuando trata del nivel superior, el autor menciona la educación superior de ciclo corto que corresponde a los estudios profesionales y técnicos avanzados, que poseen una duración variable y están orientados al mercado laboral (en el sistema español no hay una equivalencia bien definida); la educación superior de grado corresponde a los estudios universitarios, los estudios profesionales y técnicos y presenta una duración mínima de tres o cuatro años - sobre este nivel, con el Plan Bolonia, España optó por grados de cuatro años, evitando la tendencia general de Europa que es de tres años.

La educación superior de máster es otro nivel y corresponde a estudios con una duración mínima de uno o dos años - es el ciclo preparatorio del doctorado. En base a aquel plan, España optó por un año de duración (equivalente a 60 créditos), mientras que la mayoría de los países europeos establecían másteres de dos años (120 créditos). En cuanto a la educación superior de doctorado, que corresponde a los estudios que conducen a la búsqueda avanzada (la titulación de doctor se obtiene tras defender públicamente una tesis, resultado de una investigación original), no hay una duración determinada instaurada.

Práxis Educativa, Ponta Grossa, v. 11, n. 2, p. 542-546, maio/ago. 2016 Disponível em: <http://www.revistas2.uepg.br/index.php/praxiseducativa $>$ 
Dicha organización corresponde a los objetivos políticos comunitarios de la Unión Europea para 2020; además, delimita también que el análisis, en todos los estudios internacionales, respecto a la educación, esté centrado en áreas de conocimiento o materias curriculares. De ahí, el autor hace un recorte en la educación básica y centra su análisis en la compresión lectora. Él reflexiona que, dentro del panorama actual, sería necesario incluir la competencia lectora en un marco más amplio de las capacidades comunicativas, es decir, que ningún país propuso una asignatura de comunicación en la que se reconocieran y potenciaran los códigos comunicativos actuales: alfabéticos, digitales, audiovisuales, etc. (PRATS, 2013, p. 36). Prats considera que la escuela carga una fuerte dependencia de expresión de modernidad y de progreso, así como de afirmación nacional, donde la lengua materna suele ser considerada uno de los conocimientos más relevantes.

La segunda parte del estado de la cuestión, "Desempeños académicos: la excelencia en los resultados" evidencia de modo sucinto los resultados académicos del panorama europeo, específicamente de comprensión lectora, tema tratado por el informe PISA de 2009 (donde se encuentran los valores medios de los estados europeos, incluyendo Cataluña). En este sentido, el autor considera conveniente analizar estos datos con mucho detalle pues las encuestas PISA son muestras de la realidad. Dicha muestra evidencia, en datos absolutos, que Finlandia ocupa una posición destacada (la tercera a nivel mundial, después de Shanghái y Corea del Sur) y que, en términos generales, el nivel social y el estatus económico del alumno pueden influir en el rendimiento escolar cuando la escuela no reequilibra las posibles desigualdades de origen. En este sentido, el autor apunta a la necesidad de buscar más las tendencias subterráneas que los síntomas superficiales ya que es más productivo identificar las razones o las funciones de la lectura en un mundo altamente digitalizado que demostrar la capacidad de lectura de los encuestados.

En cuanto a la tercera parte, "Resultados de la educación: claves interpretativas", Prats presenta un análisis puntual de los mecanismos de evaluación - en este caso, las pruebas externas de competencias básicas en educación primaria de Cataluña en las que se evalúa el catalán, el castellano, una lengua extranjera y las matemáticas, como obligatorias y universales; y de la repetición de curso y éxito escolar, incidiendo en un aspecto nuclear que suele quedar apartado de los estudios comparados como es el concepto de permeabilidad de los sistemas educativos.

Éste es un concepto relevante en la obra del autor y se refiere a la capacidad de la sociedad para validar y acreditar los conocimientos y las competencias personales y profesionales de la trayectoria de cualquier persona. Sin embargo, la cuestión de fondo es encontrar un sistema que dé una idea más precisa e integral de los niveles de formación real de estas personas.

En el segundo capítulo del libro, "Causas y razones: los procesos educativos", el autor explica que los sistemas educativos presentaron un crecimiento significativo en los últimos cien años. Sin embargo, este crecimiento no fue acompañado de otra revolución en los métodos y las estructuras de la escuela como agente principal de la educación formal. Se nota la ampliación considerable de los márgenes de la educación obligatoria, pero se han mantenido unas estructuras educativas que, salvo algunas excepciones, no se han modificado mucho durante este último siglo. Uno de los puntos críticos de los sistemas educativos formales es la educación secundaria los países nórdicos empezaron reformas profundas en este nivel educativo hace dos décadas y, actualmente, su modelo de educación es un referente para el resto de los países europeos.

La organización del tiempo lectivo en Europa evidencia una gran variabilidad y seguramente es uno de los elementos de más complicada comparación, a escala general. Según Prats (2013, p. 113), Holanda es el país que más horas destina a la educación obligatoria (cerca de 1.000 horas anuales). Y en la inversión en recursos en la educación básica, otro elemento de

Práxis Educativa, Ponta Grossa, v. 11, n. 2, p. 542-546, maio/ago. 2016 Disponível em: <http://www.revistas2.uepg.br/index.php/praxiseducativa > 
PRATS, Enric. La educación, una cuestión de estado. Una mirada a Europa...

comparación, Dinamarca presentó, en 2009, los resultados más elevados, con cerca de un $9 \%$ de inversión sobre su PIB, en términos relativos, mientras que España se situó en el 5,01\%.

Ya en el tercer capítulo, "Principios y horizontes de la educación: una visión prospectiva", el autor menciona que los dos retos principales de la educación escolar de los últimos tiempos son la diversidad y la tecnología - el profesorado tiene ante sí a un alumnado con múltiples y variadas inquietudes y necesidades. Además, hay que considerar también los cambios y el proceso de globalización; en este sentido, las reformas educativas en Europa evidencian una preocupación por estos nuevos horizontes ya que sus documentos reflejan una orientación hacia la formación de personas capaces de competir en un mundo globalizado. Con el horizonte de 2020, la Comisión Europea lanzó una estrategia, en el año 2010, para tratar de encauzar la situación y sobreponerse así a los primeros efectos de la crisis.

En el cuarto capítulo, "Fragmento: a propósito de un despropósito", el autor hace referencia a las reformas educativas que sugieren la puesta en marcha de modelos antiguos, revestidos de nuevos. Prats afirma que España refleja progresos y retrocesos en la educación y que, desde la década de 1980 con los primeros gobiernos nacionalistas en Cataluña y socialistas en España, la educación escolar ha sufrido los titubeos que han marcado los cambios políticos. Por ello, de momento se asiste a una nueva ola reformista en España por parte de un gobierno conservador que tiene más aires de contrarreforma, y quizás de restauración, con la pretensión de recuperar y reinstalar valores caducos como la ley orgánica para la mejora de la calidad educativa - LOMCE 08/2013, que resultó en la eliminación del currículo de la asignatura de Educación para la Ciudadanía y, a su vez, puso más peso en la asignatura de Religión. Este retroceso en la formación de ciudadanos responsables contribuye a satisfacer compromisos ideológicos con una parte de la sociedad española.

En este mismo capítulo, el autor presenta las consideraciones finales que caminan en el sentido de que los fenómenos educativos se deben estudiar y valorar en función del marco contextual y desde una posición intelectual sincera que reconozca los referentes ideológicos de partida. Pues no se puede olvidar la existencia de modelos de éxito que saben acoger a todos en la escuela, hasta el final de la educación obligatoria y con resultados académicos favorables sin excluir a nadie. Modelos que desarrollan dispositivos de permeabilidad que validan la formación y la hacen más polivalente y que destinan recursos económicos a la formación del profesorado y a la investigación y la innovación pedagógica. Prats menciona que el desafío de su libro fue estudiar algunos de los mecanismos utilizados en Europa para abordar algunas de las problemáticas de la educación, que no son más que una muestra, sin duda parcial, de la realidad escolar. Pues, en la mayoría de los países del entorno, se ha alcanzado un alto nivel de escolarización a edades tempranas (educación infantil) y en etapas medias (secundaria básica) y se ha abierto la escuela a nuevas realidades, especialmente a partir de las nuevas tecnologías y también en la atención a la diversidad.

En la mayoría de los países occidentales, la escuela es todavía uno de los pocos espacios públicos donde se practica un alto nivel de tolerancia y se predica la necesidad de crear hábitos saludables de convivencia, esto se hace notar en los programas educativos y en el profesorado que apuesta por modelos inclusivos (al menos en el plano teórico). Pero cuando aumentan las interferencias indebidas sobre el sistema escolar, el profesorado retrocede y puede llegar a adoptar posiciones más conservadoras que defienden modelos selectivos y elitistas, alejados de cualquier pretensión equilibradora de las desigualdades.

Prats (2013) concluye que los estudios internacionales ofrecen una diversidad de datos demasiado descriptivos - hay necesidad de interpretar adecuadamente el porqué de las cosas y

Práxis Educativa, Ponta Grossa, v. 11, n. 2, p. 542-546, maio/ago. 2016 Disponível em: <http://www.revistas2.uepg.br/index.php/praxiseducativa > 
conocer a qué caminos llevará este proceso, sobre todo cuando se intenta construir una realidad nacional integrada en un marco europeo.

Las contribuciones de Prats además de presentar el concepto de permeabilidad (un avance para los estudios sobre el proceso de certificación de saberes y experiencias) sirven como fundamento básico para quien desea conocer la organización del sistema educativo europeo; puesto que centra la discusión en la complejidad para medir los resultados de la educación solo con la mera aplicación de pruebas estándares. Hay que reconocer la necesidad de participación de otros protagonistas en dicho proceso (el profesorado, por ejemplo) y, de la misma manera, hay que comprender los estudios comparados más allá de dos posicionamientos: copia o rechazo de un modelo. Es necesario entender las singularidades de cada uno de los países de análisis, el proceso que ellos recogieron para lograr los éxitos, y así ampliar las perspectivas de comprensión de la misma problemática que acaso esté ocurriendo en otro contexto. 